\title{
Unusual Stone Axes
}

By Bruce A. McCorquodale, Saskatchewan Museum of Natural History.

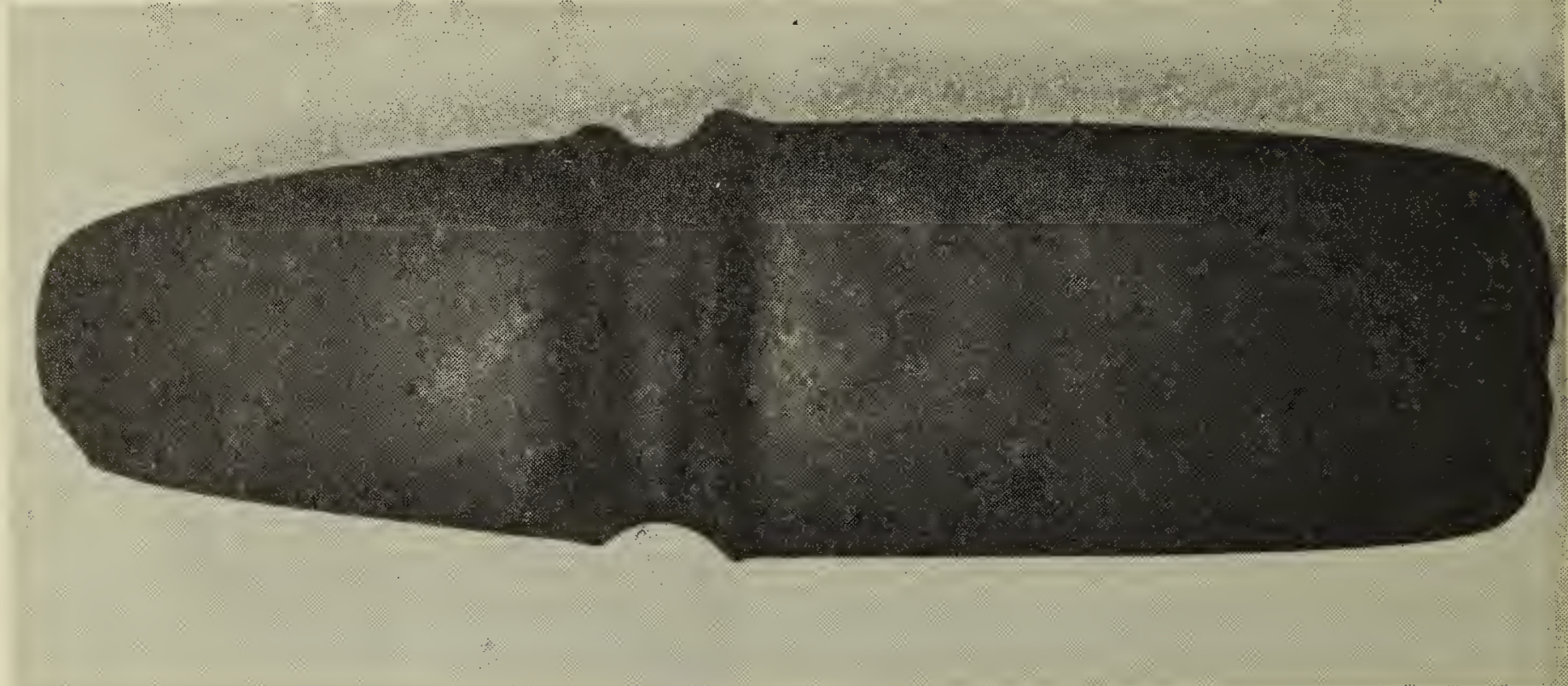

BROADVIEW AXE

Photo by R. W. Fyfo

The above illustration shows an unusual stone axe which was found in 1955 in a field (NW 1/4-35-15-6W2) by Mr. Sid Fiathers, Broadview, Sask. Mr. Father's courtesy and consideration in loaning the axe to the Museum made it possible to photograph it (see Blue Jay, Vol. 16:135 and back cover). The author is indebted to Leslie Beck, Department of Mineral Resources, for assistance in the analysis of the rock composition.

The material from which this artifact has been made is a dense, highly consolidated and slightly metamorphosed sandstone to which the name "greywacke" can be arbitrarily assigned. Field stone or greywacke occur in the glacial drift in the eastern half of Saskatchewan. Greywacke probably represents a sediment of Pre-Cambrian age and must have originated in a formation in an unknown locality in northern Manitoba or northern Saskatchewan. This material seems to be well suited to production of large artifacts. It has an amorphous structure with little or no indication of planes of fracture. It is dense, fine grained and soft enough to be easily shaped by pecking or grinding but hard enough to resist breakage and erosion. It readily absorbs greases and oils so that ordinary handling of the material will change its normal grey colour to black. Because of the black-stained surface of this axe it is easily mistaken for a basic volcanic rock.

This specimen exhibits damage only in the form of one large chip removed from the upper end and four small chips removed from the lower or cutting end. Although it may appear to be double-bitted in the illustrations, only the longer end has been sharpened. The smaller end has been smoothly rounded on its edge suggesting that its prime function may have been to provide balance to the axe. For the purposes of this description I have designated the smaller end from the flanges outward the "poll," and the other end from the flanges outward the "bit."

The axe has a highly polisned surface everywhere except in the groove between the flanges. In the groove there is indication of only minor polish on a coarse surface texture which suggests that pecking was the technique used in shaping the groove, as well as the whole axe. Prominent areas of polish within the groove, most noticeable near the larger notch, probably evidence movement of the axe within its handle or binding. The larger size and lack of confluence with the groove suggest that the concentric notch (uppermost in illustration) accommodated the end of a handle. This is substantiated by a pronounced flattening of the face of this notch. 
Comparative Dimensions (in $\mathrm{mm}$.) of Broadview and Erickson Axes

\begin{tabular}{|c|c|c|}
\hline & $\begin{array}{l}\text { Broadview } \\
\text { Specimen }\end{array}$ & $\begin{array}{c}\text { Erickson, Man. } \\
\text { Specimen }\end{array}$ \\
\hline - & 316.0 & $\begin{array}{c}126.0 \\
\text { (incomplete) }\end{array}$ \\
\hline Maximum thickness (apex of upper flange)....... & 29.0 & 34.5 \\
\hline Maximum thickness of bit (base of flange) ....... & 19.5 & \\
\hline Maximum thickness of poll (base of flange) ....... & 19.5 & 22.0 \\
\hline Maximum width (apex of lower flange) ............. & 96.0 & 117.0 \\
\hline Maximum width of bit (base of flange) ................. & 92.5 & \\
\hline Maximum width of poll (base of flange) .............. & 85.5 & 84.0 \\
\hline 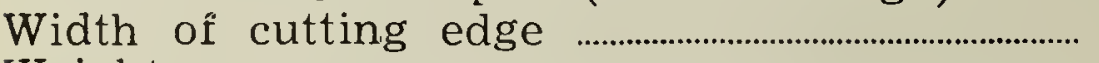 & 73.0 & \\
\hline Weight & 1093 grams & \\
\hline
\end{tabular}

\section{RARE FLUTED POINT FOUND}

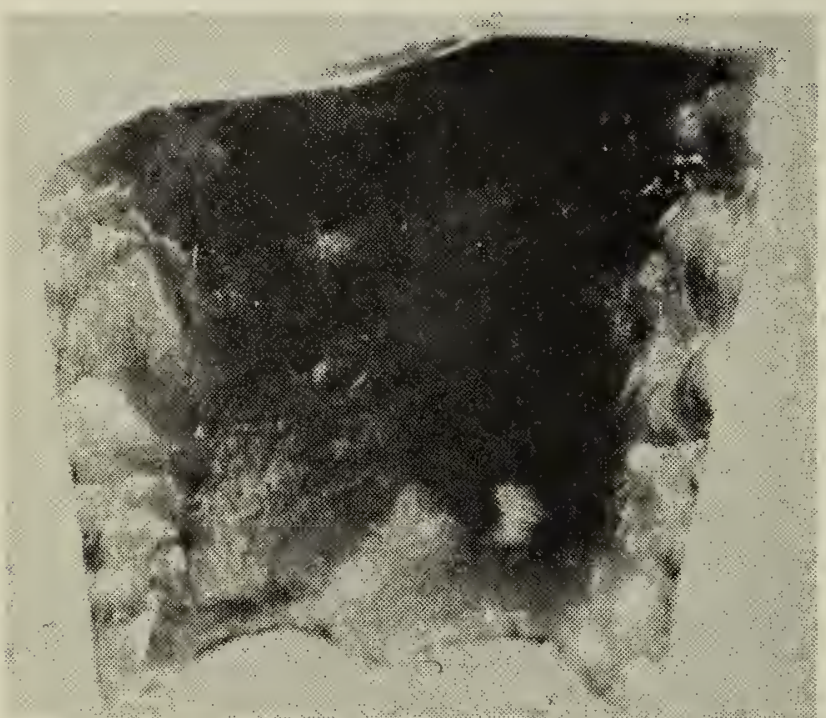

The above photographs show the two sides of a basal fragment of a fluted projectile point of the classic Folsom type ( $2 \times$ natural size). This was recently submitted to the $\mathrm{Mu}-$ seum for examination by Stanley M. Durr, Bromhead, Saskatchewan. It was found by Mr. Durr on the surface in the Bromhead district.

The point is made from brown chalcedony (Knife River flint); the edges of the base and sides are heavily ground. An extremely skilfull and

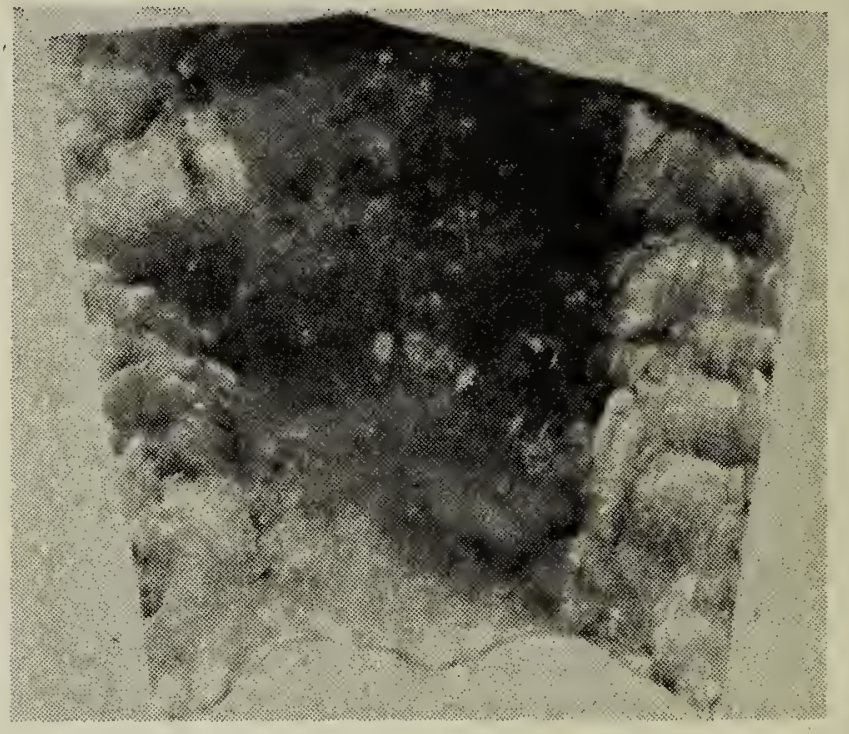

Photos by R. W Fyfe

well-controlled technique of flintworking is evident. One cannot but admire the artistry of the early hunters of perhaps 10,000 years ago who produced these scarce artifacts.

A few cther fluted points have been described in the Blue Jay (Vol. 16: 42-43) and the Museum has records of fluted points from Jameson, Kamsack, McCord, and Weyburn.Robert W. Nero, Saskatchewan Museum of Natural History.

\section{ARCHAEOLOGICAL POSITION ANNOUNCED!}

Word has just been received that the Museum has been granted approval of its request for a curatorial position in archaeology and ethnology. The position is already being advertised and it is hoped that an appointment may be made after January 1, 1959. This is an important event in the development of the $\mathrm{Mu}$ seum since this is the first official research position to be established.

This augurs well for the acceptance of our Museum as a scientific institution. We look forward with anticipation to an orderly development of archaeological research. It seems highly likely that this programme will yield considerable information about the prehistery of mankind in the Northern Plains. Archaeologists ihroughout North America have already expressed a strong interest in the three scientific excavations which have been conducted in Saskatchewan. Dr. H. Wormingiton has suggested that this region may hold the key to the prehistory of the Plains: Congratulations to the Museum and the Department of Natural Resources. - Editor. 